\title{
Rapid determination of small molecule pollutants using metal-organic frameworks as adsorbent and matrix of MALDI-TOF-MS
}

\author{
Saihua Wang a, b, Hongyun Niu ${ }^{\text {a }}$, Tao Zeng ${ }^{\mathrm{c}}$, Xiaole Zhang ${ }^{\mathrm{d}}$, Dong Cao ${ }^{\mathrm{a}}$, Yaqi Cai ${ }^{\mathrm{a}, \mathrm{b},{ }^{*}}$ \\ a State Key Laboratory of Environmental Chemistry and Ecotoxicology of Research Center for Eco-Environmental Sciences, Chinese Academy of Sciences, \\ Beijing, 100085, China \\ ${ }^{\mathrm{b}}$ University of Chinese Academy of Sciences, Beijing, 100049, China \\ c College of Environment, Zhejiang University of Technology, Hangzhou, Zhejiang Province, 310032, China \\ d College of Life Science, North China University of Science and Technology, Tangshan, 063000, Hebei Province, China
}

\section{A R T I C L E I N F O}

\section{Article history:}

Received 25 March 2016

Received in revised form

21 October 2016

Accepted 21 October 2016

Available online 22 October 2016

\section{Keywords:}

Metal-organic framework

MALDI-TOF-MS

PFOS

Matrix

Enrichment

\begin{abstract}
A B S T R A C T
Matrix-assisted laser desorption/ionization time-of-flight mass spectrometry (MALDI-TOF-MS) has been commonly used for the determination of macromolecules owing to the high sensitivity and convenience. However, the direct analysis of small molecule with MALDI-TOF-MS remains a challenge due to the matrix interference in the low mass region. In this paper, metal-organic frameworks (MOFs: MOF-5, MOF-235, Cu-btc MOFs and Uio-66(Zr)-2OH) were successfully used as both adsorbent and significant matrix of MALDI-TOF-MS method for the analysis of small molecule pollutants. Benzo(a)pyrene (BaP) and perfluorinated compounds (PFCs) were chosen as model analytes to investigate the matrix function of MOFs with LDI measurement in positive and negative reflection mode, respectively. As a result, MOFs exhibit high laser desorption/ionization efficiency and function well in both positive-ion and negativeion modes. Compared to the MS spectra with conventional matrices, MS spectra obtained on MOFs matrix are only featured by deprotonated molecule ion peaks without background interference. Furthermore, MOFs, with large surface area and $\pi$ - $\pi$ stacking structure, can also enrich trace amounts of small molecule pollutants from solution efficiently and quickly. According to these, we developed a simple method in which MOFs-solid-phase extraction (SPE) enrichment was combined with MALDI-TOFMS to realize the direct analysis of perfluorooctanesulfonate (PFOS) in real water samples.
\end{abstract}

() 2016 Elsevier Inc. All rights reserved.

\section{Introduction}

Matrix-assisted laser desorption/ionization has been one of the most commonly used ionization methods since it appeared in the later 1980s owning to its advantages of short-time experimental cycle, simple pretreatment procedure, low consumption of samples and high sensitivity [1-4]. Based on it, matrix-assisted laser desorption/ionization time-of-flight mass spectrometry (MALDITOF-MS) is an accurate and powerful technique for the analysis of big molecules such as peptide, protein and nucleic acid [5-7]. In this technique, MALDI works on the mechanism of laser irradiation of a sample incorporated into a UV-absorbing matrix, leading to the desorption and ionization of analytes which is detected by using

\footnotetext{
* Corresponding author. State Key Laboratory of Environmental Chemistry and Ecotoxicology of Research Center for Eco-Environmental Sciences, Chinese Academy of Sciences, Beijing, 100085, China.

E-mail address: caiyaqi@rcees.ac.cn (Y. Cai).
}

TOF-MS subsequently. The laser desorption/ionization process and the reproducibility of signal intensity is generally matrix dependent. Since conventional organic matrices such as $\alpha$-cyano-4hydroxycinnamic acid (CHCA) and 2,5-dihydroxybenzoic acid (DHB) usually break up under laser irradiation and produce highintensity signal interference in the low molecular-weight range, the application of MALDI in the measurement of small molecules ( $\leq 500 \mathrm{Da}$ ) was greatly limited $[8,9]$.

To realize the analysis of small molecules with MALDI-TOF-MS, various new organic matrices with low background and new ingenious matrix-free platforms by using various micro/nanostructures have been developed to eliminate the interference of matrix-related ions. For instance, nanomaterials, including metal materials (Co, Au, Ag, and Pt) [5,10], metal oxides [11-13], silicon nanoparticles [14] and carbon based materials (e.g., graphite, carbon nanotubes, fullerene, graphene) [15-21] have all been adopted for assisting the laser desorption/ionization (LDI) of low-molecularweight analytes such as glucose, creatinine, and amino acids et al. 
These materials produce little background signal of less than $500 \mathrm{Da}$ and widen the application field of MALDI into the detection of small molecules. However, these matrices reveal low solubility and dispersibility in solution that causes the heterogeneous crystallization with the analytes, and this would lead to relatively lower sensitivity and shot-to-shot reproducibility compared with the conventional matrixes. Therefore, it is necessary to explore new matrixes for the detection of small molecules by LDI measurement with high sensitivity, reproducibility and stability.

Metal-organic frameworks (MOFs) are a large group of highly porous crystals in which metal ions are connected with organic linker molecules to fabricate two-or three-dimensional structures. They have attracted considerable attention because of their potential for a wide range of applications in separation, proton conductivity, gas storage, drug delivery, heterogeneous catalysis and luminescent sensors [22-26]. The $\pi-\pi$ structure of the organic linker in MOFs such as terephthalic acid (TPA) and benzenetricarboxylic acid (BTC) $[27,28]$ and the oxygenated function groups (- $\mathrm{COOH}$ and $-\mathrm{OH})$ help to absorb the energy from UV laser radiation and transfer energy to small molecule pollutants, which facilitates the desorption/ionization of analytes [29]. Moreover, MOFs also exhibit high adsorption ability toward small organic molecules owing to the large surface area and $\pi-\pi$ stacking structure and can be dispersed in solution uniformly. Therefore, MOFs could be employed in analyzing environmental pollutants with LDI-TOF-MS measurement as both adsorbent and matrix.

Herein, four different metal-organic frameworks (MOFs: MOF-5, MOF-235, Cu-btc MOFs and Uio-66(Zr)-2OH) were easily prepared and used as LDI matrices for the analysis of small molecule pollutants. The desorption/ionization efficiencies of the four matrices were evaluated in both positive and negative reflection mode. In addition, MOFs were further used as solid-phase extraction (SPE) adsorbent for the concentration of perfluorinated compounds (PFCs) from water samples, followed by the direct MALDI-TOF-MS analysis. This work, combining the MOFs-SPE enrichment with MALDI-TOF-MS measurement, was expected to provide a rapid and efficient method for the determination of small molecules.

\section{Experimental}

\subsection{Chemicals and materials}

Ferric chloride hexahydrate $\left(\mathrm{FeCl}_{3} \cdot 6 \mathrm{H}_{2} \mathrm{O}\right)$, copper nitrate $\left(\mathrm{Cu}\left(\mathrm{NO}_{3}\right)_{2} \cdot 3 \mathrm{H}_{2} \mathrm{O}\right)$, zinc acetate dehydrate $\left(\mathrm{Zn}(\mathrm{OAc})_{2} \cdot 2 \mathrm{H}_{2} \mathrm{O}\right), \mathrm{N}, \mathrm{N}-$ dimethylformamide (DMF) were obtained from Sinopharm Chemistry Reagent Co., Ltd. (Beijing, China). Zirconium chloride $\left(\mathrm{ZrCl}_{4}\right), 1,3,5$-benzenetricarboxylic acid (99\%, BTC) and $p$-chloranil were purchased from J\&K Chemicals Ltd. (Beijing, China). Terephthalic acid (99\%, PTA) and 2, 5-dihydroxyterephthalic acid (DHTA) were from Acros Organics (New Jersey, USA). Benzo(a)pyrene (BaP) was supplied by AccuStandard (New Haven, USA) and dissolved in acetonitrile to acquired concentration. Humic acid (HA), bovine serum albumin (BSA), Perfluorobutyric sulfonate (PFBS), perfluorohexane sulfonate (PFHxS), perfluorooctanic sulfonate (PFOS), tetrabromobisphenol A (TBBPA), tetrachlorobisphenol A (TCBPA), bisphenol A (BPA), 2, 4, 6-tribromophenol (TBP) and ethinylestradiol (EE2) were offered by Sigma-Aldrich (Steinheim, Germany). Pentabromophenol (PBP), pentachlorophenol (PCP), 3, 5, 3'-triiodothyronine (T3) and tributoxyethyl phosphate (TBEP) were from Dr. Ehrenstorfer (Augsburg, Germany). $\alpha$-Cyano-4hydroxycinnamic acid (CHCA) and 2, 5-dihydroxybenzoic acid (DHB) were provided by Bruker Doltonics Corp (Germany). HPLCgrade acetonitrile was from Fisher Scientific (NJ, USA). All chemicals were of analytical reagent grade and used without further purification. Ultrapure water was prepared in the lab by using a Milli-Q SP reagent water system (Millipore, Bedford, MA, USA).

\subsection{Instrument}

The morphologies of the synthesized materials were surveyed by a transmission electron microscopy (TEM, Hitachi, Japan) operating at $80 \mathrm{kV}$ accelerated voltage. FTIR spectra were recorded using $\mathrm{KBr}$ pressed pellets on a NEXUS 670 Infrared Fourier Transform spectrometer (Nicolet Thermo, USA). $\mathrm{N}_{2}$ sorption analysis was carried out by a volumetric adsorption analyzer (Autosorb ${ }^{\mathbb{R}}-1$, Quantachrome $^{\circledR}$, America) at $77 \mathrm{~K}$. The specific surface area was calculated according to the Brunauer-Emmett-Teller (BET) method. The pore size distribution (PSD) plot was obtained based on the HK model.

\subsection{Preparation of MOFs}

\subsection{1. $M O F-5$}

Firstly, $0.506 \mathrm{~g}$ terephthalic acid was dissolved in $40 \mathrm{~mL}$ DMF and $1.70 \mathrm{~g} \mathrm{Zn}(\mathrm{OAc})_{2} \cdot \mathrm{H}_{2} \mathrm{O}$ was dissolved in $50 \mathrm{~mL}$ DMF. Then, the obtained zinc salt solution was added to the terephthalic acid solution dropwise under magnetic stirring and white precipitate appeared immediately. The mixture solution was stirred for another $2 \mathrm{~h}$ and the precipitate was afterwards separated, rinsed with DMF and distilled water, and dried at $50{ }^{\circ} \mathrm{C}$ for $12 \mathrm{~h}$ to yield MOF-5 [30].

\subsubsection{Cu-btc MOFs}

To prepare $\mathrm{Cu}$-btc MOFs, $1.82 \mathrm{~g}$ copper nitrate $\left(\mathrm{Cu}\left(\mathrm{NO}_{3}\right)_{2} \cdot 3 \mathrm{H}_{2} \mathrm{O}\right)$ and $0.875 \mathrm{~g} \mathrm{1,3,5-benzenetricarboxylic}$ acid were dissolved in $50 \mathrm{~mL}$ methanol under ultrasonication, respectively. Then the copper nitrate solution was transferred into the tricarboxylic acid solution under vigorous stirring. The mixture solution was kept at room temperature for $2 \mathrm{~h}$ until MOF precipitation finished. The precipitate was obtained by centrifugation and washed with methanol twice. Finally, the blue powder of Cu-btc MOFs was dried in vacuum at $50{ }^{\circ} \mathrm{C}$ for $12 \mathrm{~h} \mathrm{[31].}$

\subsection{3. $\mathrm{MOF}-235$}

MOF-235 was synthesized through a solvothermal reaction according to the previous report [32]. Briefly, $0.20 \mathrm{~g} \mathrm{FeCl}_{3} \cdot 6 \mathrm{H}_{2} \mathrm{O}$ and $0.205 \mathrm{~g}$ terephthalic acid were dissolved in $60 \mathrm{~mL}$ DMF and then mixed with $60 \mathrm{~mL}$ ethanol. The mixture solution was transferred into a Teflon-lined autoclave and heated at $80{ }^{\circ} \mathrm{C}$ for $24 \mathrm{~h}$. The product was washed with DMF-ethanol mixture solution for three times and dried in vacuum.

\subsection{4. $\operatorname{Uio66}(\mathrm{Zr})-2 \mathrm{OH}$}

A mixture of $0.932 \mathrm{~g} \mathrm{ZrCl}_{4}$ and $0.792 \mathrm{~g} \quad 2$, 5dihydroxyterephthalic acid in $40 \mathrm{~mL}$ DMF was sealed in a Teflonlined autoclave and heated to $130{ }^{\circ} \mathrm{C}$ for $24 \mathrm{~h}$. After cooling down to room temperature, the precipitates were obtained by centrifugation and then activated by DMF and methanol for $12 \mathrm{~h}$, respectively. Finally, the product was washed with DMF and ethanol, and then dried in vacuum for $12 \mathrm{~h}$.

\subsection{MALDI-TOF-MS analysis}

In a typical MALDI-TOF-MS analysis, $10 \mu \mathrm{L}$ of the matrix solution and $10 \mu \mathrm{L}$ of analyte solution were mixed by vortex in a centrifuge tube, followed by directly doting $1 \mu \mathrm{L}$ of the matrix-analyte mixture onto the 384-well MALDI target plate (AnchorChip TM target plate, provided by Bruker Doltonics, Germany), and air-dried for further MALDI-TOF-MS analysis. 
All mass spectra were performed on an Autoflex III MALDI-TOFMS (Bruker Daltonics, Germany) equipped with a pulsed nitrogen laser (wavelength $337 \mathrm{~nm}$ ) for irradiation of analytes and an accelerating voltage from $-20 \mathrm{kV}$ to $20 \mathrm{kV}$. Mass spectra in both positive and negative reflection mode were acquired with 200 laser shots with a LeCroy 9314 digital oscilloscope. All mass spectra were analyzed by Flex Analysis software provided by Bruker Daltonics Corp.

\subsection{Enrichment and analysis of PFOS from real water samples}

The enrichment procedure of PFOS was as follows: $0.5 \mathrm{mg}$ of the MOFs was added into $10 \mathrm{~mL}$ of water solution spiked with stock solution of PFOS, the mixture was then vibrated for $30 \mathrm{~min}$. The MOFs with the sorbed analytes were isolated by centrifugation and redispersed in $20 \mu \mathrm{L}$ of methanol, and $1 \mu \mathrm{L}$ of the MOFs-target conjugate was pipetted onto the stainless steel plate for the MALDI analysis.

River water and tap water samples were filtered through $0.45 \mu \mathrm{m}$ nylon filter. The following procedure was the same to the above.

\section{Results and discussion}

\subsection{Characterization of MOFs}

The morphology of the as-prepared MOFs was characterized by TEM. As shown in Fig. 1, the obtained MOF-5 possesses a clear cubic architecture with a mean diameter of $1 \mu \mathrm{m}$. The $\mathrm{Cu}-$ btc MOFs and MOF-235 particles are relatively uniform and appear in octahedral shape with an average edge length of $0.75 \mu \mathrm{m}$ and $0.9 \mu \mathrm{m}$, respectively. The Uio66(Zr)-2OH has a homogeneous spherical structure with a diameter of $1.8 \mu \mathrm{m}$.

$\mathrm{N}_{2}$ adsorption/desorption isotherms of MOFs (Fig.S1) show high adsorption at low pressure and exhibit a sharp increase at relatively low pressure $\left(\mathrm{P} / \mathrm{P}_{0}\right)<0.02$, suggesting the presence of microporous structure of MOFs. The textural properties of MOFs are given in Table S1. The surface area and pore size of the MOFs are ranging

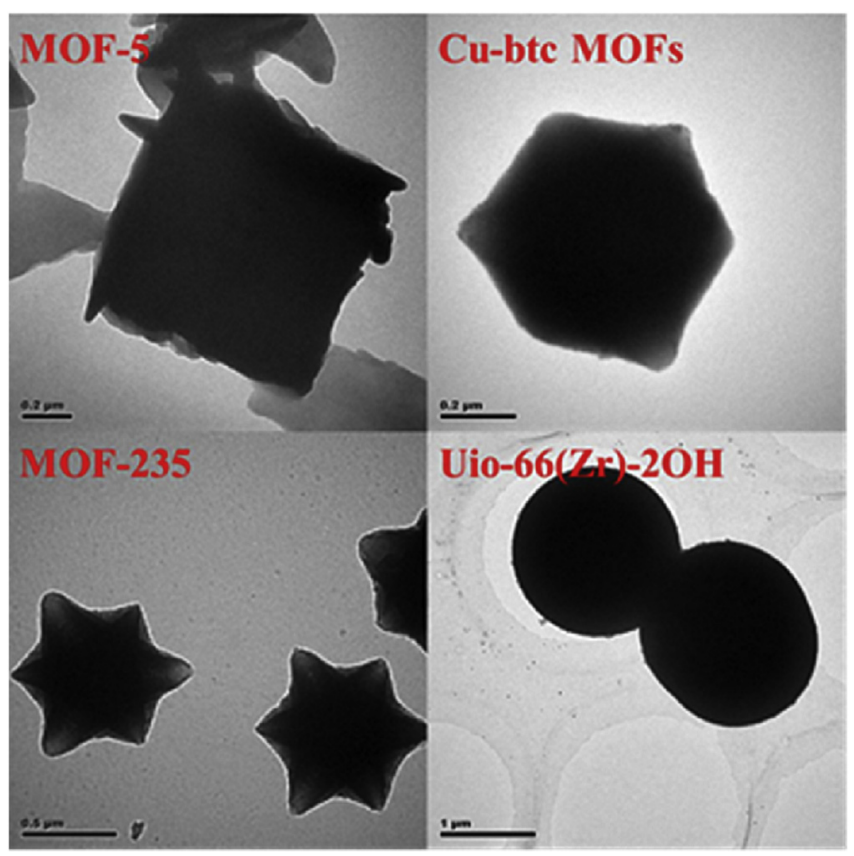

Fig. 1. TEM images of MOFs. from 918 to $1450 \mathrm{~m}^{2} \mathrm{~g}^{-1}$ and $5.9-10.3 \AA$, respectively. All of the MOFs possess high surface and micro scaled pore size.

According to the FTIR spectra of the synthesized products (Fig.S2A), all samples demonstrated strong peaks in the region of $1300-1650 \mathrm{~cm}^{-1}$, which are attributed to the $\mathrm{C}=\mathrm{O}$ in carboxylates, $\mathrm{C}=\mathrm{C}$ in aromatic compound of organic linker, and $\mathrm{C}-\mathrm{O}$ bond of $\mathrm{C}-$ $\mathrm{OH}$ group of carboxylic acid inside the molecules, respectively. This indicated that the main functional groups on PTA or BTC organic linker have been kept for all MOFs. The peaks at around $3400 \mathrm{~cm}^{-1}$ are ascribed to the hydroxyl groups which are generated from the surface-adsorbed water by MOFs and the width of the peak is related to the intensity of the hydrogen bond. Uio-66( $\mathrm{Zr})-2 \mathrm{OH}$ corresponds to a wider peak of hydroxyl group suggesting the existing of hydroxyl group in its ligand.

\subsection{MOFs as MALDI matrix in positive reflection mode}

UV-Vis spectra of MOFs were recorded to investigate the capacity of MOFs to absorb ultraviolet rays. As shown in Fig.S2B, all the obtained MOFs have absorption bands around $337 \mathrm{~nm}$, which endows them with the possibility of absorbing laser energy and transferring energy. Furthermore, in the UV-Vis spectrum of Uio$66(\mathrm{Zr})-2 \mathrm{OH}$, an obvious adsorption peak occurs at $350 \mathrm{~nm}$, which is probably assigned to the extra hydroxyl group. Since MOFs have absorption in the near-ultraviolet region, they have great possibility to be used as matrix in the MALDI-TOF-MS method.

Benzo[a]pyrene (BaP), well known for its high toxicity and carcinogenic properties, was selected to investigate the matrix assistant function of MOFs with LDI measurement in positive reflection mode. Fig.S3 shows the mass spectra of BaP obtained by using four MOFs as the MALDI matrices. With MOFs as matrices, obvious signals of $\left[\mathrm{BaP}-\mathrm{e}^{-}\right]^{+}$appeared at 251.6 without background signal interference in the mass spectra. The strongest MS signal intensity and highest $\mathrm{S} / \mathrm{N}$ value was observed as Uio-66(Zr)-2OH was used as matrix. The effective desorption/ionization of BaP can be contributed to two main reasons. First, MOFs with relatively high surface area and abundant benzene rings skeleton could capture BaP from solution readily, which facilitates the desorption/ionization of BaP. Second, the $\pi$-conjugated networks, oxygenated functional groups, and metal cations make MOF a proton and electron donor or acceptor, which is helpful to absorb the laser energy and transfer energy to BaP, thus assist the desorption/ionization of BaP [18]. The four MOFs exhibited distinct LDI efficiencies towards BaP, which was in the order of Uio-66(Zr)-2OH > MOF-5 >Cu-btc MOFs > MOF235. But there did not have clear correlation between the surface area of MOFs and the LDI efficiencies. A possible reason for the remarkable higher LDI efficiency of BaP assisted by Uio-66(Zr)-2OH could be due to the different binding affinities of BaP toward the various MOFs. As we all know, BaP is highly hydrophobic and strongly adsorbed on the surface of carbon materials, reverse phase adsorbents and benzene-rings-rich adsorbents. The strong adsorption usually leads to inefficient desorption of BaP from adsorbents. In MALDI, potent adsorption of analytes on matrix accounts for poor LDI of analytes [33]. The functionalization of hydroxyl groups makes the surface of Uio-66(Zr)-2OH more hydrophilic than other MOFs, thus Uio-66(Zr)-2OH provides weaker interactions to $\mathrm{BaP}$, favoring the ease of phase transition/destruction of the analytes upon laser irradiation. On the other hand, the hydroxyl groups on Uio-66(Zr)-2OH might liberate protons, which then diffuse to BaP and facilitate the LDI of BaP [34].

The conventional matrices, $\alpha$-cyano-4-hydroxycinnamic acid (CHCA) and 2, 5-dihydroxybenzoic acid (2, 5-DHB), were also employed for the detection of Bap in this study. As presented in Fig. S4, BaP can be ionized by these organic matrices, but the interference signals from matrix cluster ion obstructed the analysis 
of BaP severely. Therefore, MOFs were more suitable for the LDITOF-MS analysis of BaP than the conventional matrices.

To obtain the best mass signal response, we studied the effect of matrix amount on the MALDI performance. The mass spectra of BaP with different concentrations of matrix (take MOF-5 for example) are shown in Fig. S5. We found that the BaP signal intensity increased with the increasing amount of matrix from 0.10 to $0.50 \mu \mathrm{g} \mu \mathrm{L}^{-1}$. However, the signal intensity decreased dramatically when the amount of matrix increased from 0.50 to $5.0 \mu \mathrm{g} \mu \mathrm{L}^{-1}$ because of the decreasing energy transfer efficiency at high concentration of matrix. Therefore, the optimal matrix concentration for the analysis of BaP is $0.50 \mu \mathrm{g} \mu \mathrm{L}^{-1}$.

To evaluate the sensitivity of this method, different concentrations of BaP were mixed with MOFs and dotted on the MALDI plate, followed by the determination with MALDI-TOF-MS. It was observed that the BaP peak intensity increased obviously with the increasing BaP concentration (Fig. 2). Furthermore, a linear relationship was achieved between the peak intensity and the concentration of BaP in the range of $0.5-1000 \mathrm{ng} \mathrm{mL}^{-1}\left(\mathrm{R}^{2}=0.992\right)$ and the limit of detection was calculated to be $0.21 \mathrm{ng} \mathrm{mL}^{-1}$. Contributed by the uniformed particle size and perfect dispersion of these MOFs in suspension solvent, the MS signals of analytes have good shot-to-shot and spot-to-spot reproducibility. For example, when the concentration of BaP is $500 \mathrm{ng} \mathrm{mL}^{-1}$ and MOF-5 is employed as matrix, the inner- and inter-spot relative standard deviation (RSD) of peak intensities for $\mathrm{BaP}(\mathrm{n}=10)$ is calculated to be $3.84 \%$ and $9.13 \%$, respectively. This provides a solid foundation for quantitative analysis of small molecules.

\subsection{MOFs as MALDI matrix in negative reflection mode}

Perfluorinated compounds (PFCs) are a class of man-made chemicals and have been widely used in industrial and commercial applications, including coatings for carpets and fabrics, fastfood contact materials, fire-fighting foams, hydraulic fluids and pesticides, due to their high surface activities, thermal and chemical resistances, and hydro- and lipophobic properties. PFCs have become persistent and widespread environmental contaminants with bioaccumulation properties and toxic effects to humans and organisms. Experiments have shown that PFCs could cause immune system disorders, endocrine disruption, reduced fetal growth and gestational diabetes $[35,36]$. Because of the potential hazards of PFCs to the environment and human health, it is necessary to develop fast and sensitive analytical techniques for PFCs. In this work, we chose three PFCs as analytes to study the application of MOFs as matrix of MALDI in negative desorption/ionization mode for the detection of PFCs. When MOFs were utilized as matrices, the

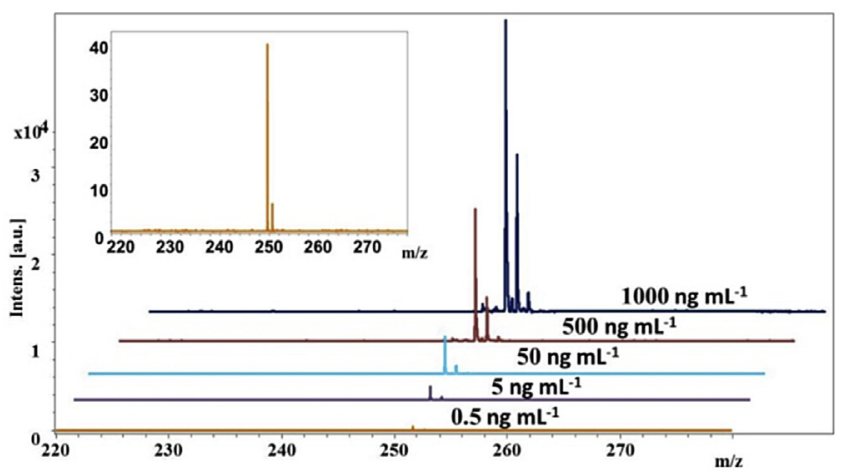

Fig. 2. MALDI mass spectrum of different amount of BaP in positive mode with MOFs as matrix. Inset shows the original mass spectrum of $0.5 \mathrm{ng} \mathrm{mL} \mathrm{m}^{-1} \mathrm{BaP}$. sulfonic group of PFOS was easily deprotonated into [PFOS-H $]^{-}$ which could be detected at 498.5 in the negative reflection mode (Fig.S6). The obvious PFOS ion peak can be discerned in all mass spectra without background signal interference, suggesting the successful deprotonation/ionization of PFOS by the four matrices. Similar to the positive mode, the optimal matrix concentration for PFOS in negative reflection mode was $0.50 \mu \mathrm{g} \mu \mathrm{L}^{-1}$ as well (Fig.S7). Likewise, only deprotonated ions of PFBS at $\mathrm{m} / \mathrm{z} 298.3$ and PFHxS at $\mathrm{m} / \mathrm{z} 398.4$ were observed in the respective mass spectra. The sensitivity of the PFCs detection with MALDI measurement was also examined and the results are shown and summarized in Fig. 3 and Table 1. The calibration curves of PFOS, PFHxS and PFBS were linear over the range of 5-1000 $\mathrm{ng} \mathrm{mL}-1$ and the detection limits (S/ $\mathrm{N}=5$ ) of them are $0.64,0.94$ and $0.85 \mathrm{ng} \mathrm{mL}-1$, respectively, demonstrating wide dynamic range of response and high sensitivity of the method.

To further evaluate the application of MOFs as matrix of MALDI, we tested the use of MOFs as matrix for detection of multiple small molecules in aqueous samples (molecular weight 228-651),
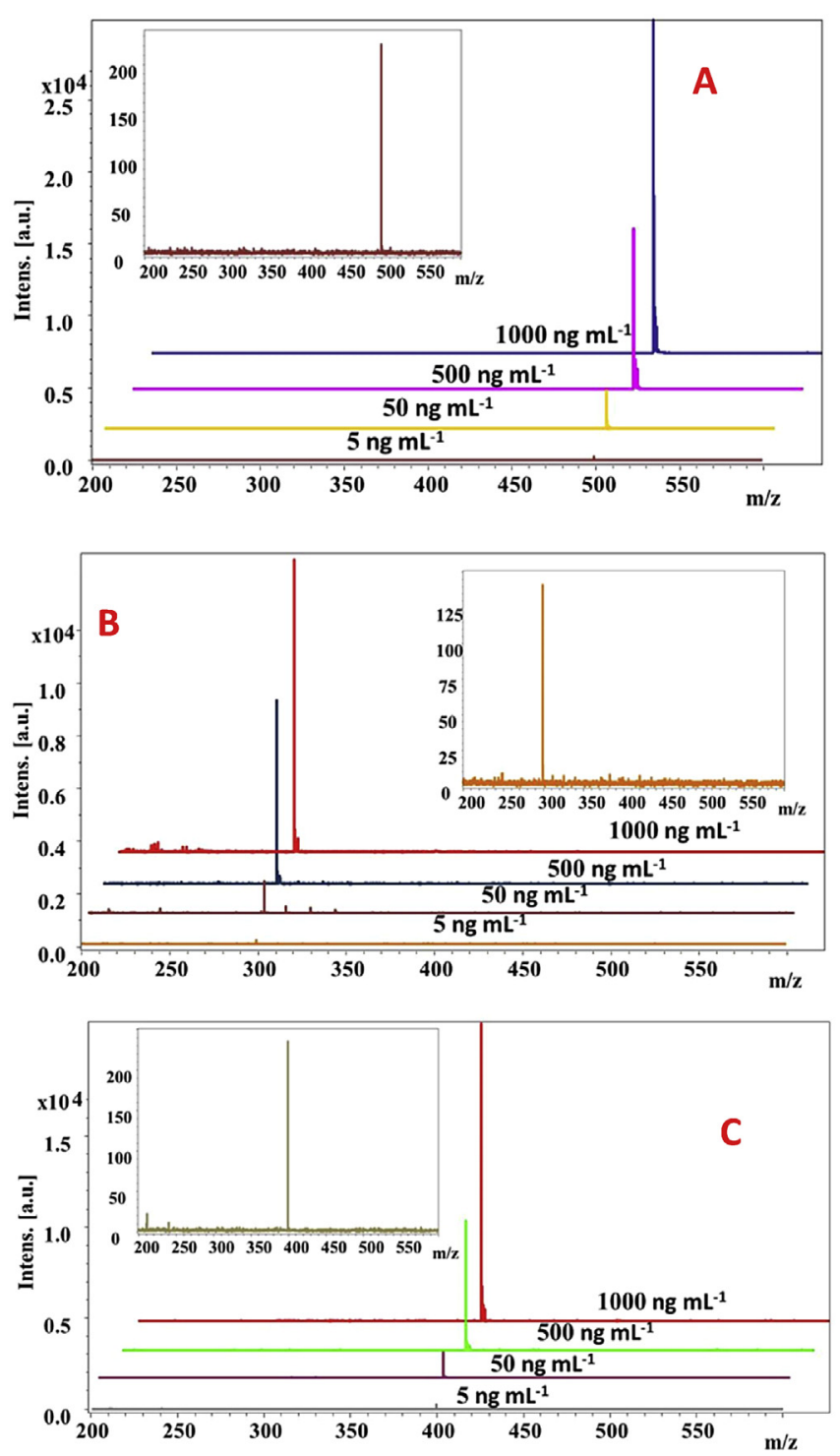

Fig. 3. MALDI mass spectrum of different amount of PFOS (A), PFBS (B) and PFHxS (C) with MOFs as matrix in negative mode. Inset shows the original mass spectrum of $5 \mathrm{ng} \mathrm{mL}^{-1}$ PFCs. 
Table 1

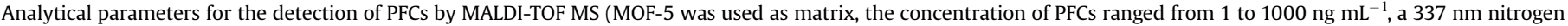
laser with the frequency of $100 \mathrm{~Hz}$ was used, and the laser power was set to $50 \%$ ).

\begin{tabular}{|c|c|c|c|c|c|}
\hline Analyte & Equation & $\mathrm{r}$ & $\operatorname{RSD}(\%)(\mathrm{n}=5)$ & Linear range (ng $\mathrm{mL}^{-1}$ ) & Detection limit $\left(\mathrm{ng} \mathrm{mL}^{-1}\right)$ \\
\hline PFOS & $\mathrm{Y}=24.1 \mathrm{x}+533.0$ & 0.996 & $0.34-9.1$ & $5-1000$ & 0.64 \\
\hline PFHxS & $Y=14.6 x+83.4$ & 0.997 & $2.6-9.9$ & $5-1000$ & 0.94 \\
\hline PFBS & $Y=11.5 x+276.4$ & 0.999 & $2.9-12$ & $5-1000$ & 0.85 \\
\hline
\end{tabular}

including six chlorophenols and bromophenols compounds (BPA, PCP, TBP, TCBPA, PBP, TBBPA), ethinylestradiol (EE2), $p$-chloranil, 3 , $5,3^{\prime}$-triiodothyronine (T3) and an organophosphate esters tributoxyethyl phosphate (TBEP). All these chemicals are widely used in industrial and commercial applications but have been proved to have potential risks to human health or ecosystems. In brief, all types of these small molecules could be detected by using MOFs as matrix. Besides ionic analytes such as PFOS, which is very easy to ionize and desorb from the matrix, mediated polar and nonpolar compounds (for instance $p$-chloranil, EE2 and TBEP) can be efficiently ionized/desorbed with the aid of MOFs matrixes (Fig.S8). For the desorption/ionization efficiency of small molecules, in the case of the signal intensity of Bap (50 $\left.\mathrm{ng} \mathrm{mL}^{-1}\right)$, the results of MOFs are equal or higher than those obtained using grapheme, carbon nanotube or inorganic matrices [18,19]. More importantly, MOFs matrix can yield significantly higher signals and achieve the ionization/desorption for nonpolar analytes. Accordingly, MOFs as MALDI matrix are of great value for the analysis of small molecule pollutants.

\subsection{MOFs as SPE adsorbent and matrix for PFOS}

MOFs, with relatively large surface area, are full of $\pi$-conjugated networks and oxygenated functional groups, which endows them with high adsorption ability to capture compounds from solution $[25,37]$. Therefore, MOFs may also serve as SPE adsorbent materials for the enrichment of trace molecules in water. PFOS was chosen as model molecule to further testify the enrichment ability of MOFs. To obtain the best mass signal response, recoveries, and reproducibility of the results, we optimized the sample pretreatment conditions, including solution $\mathrm{pH}$, amount of MOFs and suspension solvent. It was found that solution $\mathrm{pH}$ did not affect the detection of PFOS, thereby the solution $\mathrm{pH}$ was not adjusted in the following experiment. The PFOS signal intensity increased with the increasing amount of MOFs and the highest value achieved at $50 \mathrm{mg} \mathrm{L}^{-1}$ (Fig.S9A), after that, the intensity decreased with the further increase of MOFs amount, resulting from the reduced PFOS ion yield due to the low amount of adsorbed PFOS per MOF. Thus, a dosage of $50 \mathrm{mg} \mathrm{L}^{-1}$ of MOFs was selected as the optimal condition for PFOS enrichment and detection. The disperse solvent could affect the dispersibility of MOFs and the crystallization of matrix with the analytes. In this work, acetone, methanol and acetonitrile were selected as suspension agent of the MOFs with the sorbed analytes. Acetone could give rise to heterogeneous distribution of MOFs on the MALDI plate, which adversely affected the MS signals of PFOS. Both methanol and acetonitrile lead to perfect uniformity of MOFs on MALDI plate and high $\mathrm{S} / \mathrm{N}$ ratio of PFOS ion signal (Fig.S9B). Considering the toxicity of acetonitrile, methanol was employed to disperse the matrix. Under the optimal conditions, the PFOS ion signals in MALDI spectra are significantly increased after 30 min of enrichment by all the four MOFs (Figs.4 and S10). Furthermore, the PFOS could not be detected at the low concentration of $1 \mathrm{ng} \mathrm{mL}^{-1}$, whereas after enrichment, there were obvious PFOS ion signal in the mass spectra (Fig. 5). The detection limits of

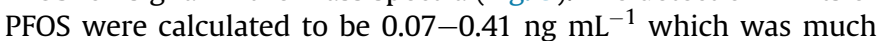
lower than those without the enrichment process (Table S1). Thus,

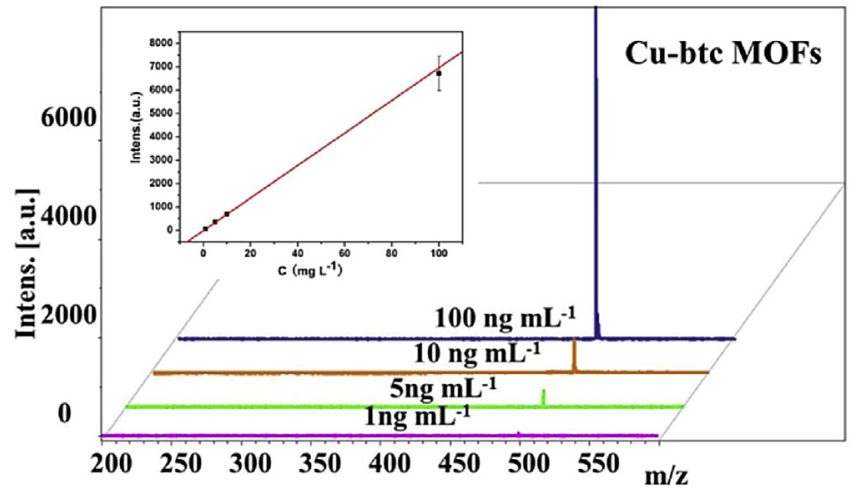

Fig. 4. Mass spectra of different concentrations of PFOS in water solution with MOFs as adsorbent and matrix, the insert is the LDI-TOF-MS response curves for increasing concentrations of PFOS.

MOFs reveal high enrichment ability and perfect LDI performance toward PFOS and can serve as both adsorbent and MALDI matrix for the detection of some small molecules.

In order to examine the anti-interference ability of the proposed strategy, we also investigated the interference of natural organic matter (HA) and protein (BSA) toward PFOS determination with this method. As shown in Fig.S11, the PFOS signals have no obvious difference after $5 \mathrm{mg} \mathrm{L}^{-1} \mathrm{HA}$ or BSA was added to the water sample, indicating that the coexistence of HA and BAS would not interfere with the detection of PFOS in real water sample.

To evaluate the feasibility of the method for the analysis of PFOS in real water sample, it was applied to the determination of PFOS in river water and tap water samples. PFOS was not detected in these real water samples. While as $5 \mathrm{ng} \mathrm{mL}^{-1}$ of PFOS were spiked into these samples, [PFOS-H $]^{-}$signal with high intensity was observed in the mass spectrum of each water sample after pretreatment with MOFs (Fig.S12). According to the established calibration curves, the recoveries of PFOS in river water ranged in $92.8-103 \%$ as different MOFs were utilized as matrix and adsorbents, and the $\operatorname{RSD}(n=3)$ of recoveries ranged from 2.7 to $10.2 \%$, displaying good method precision.

\section{Conclusions}

In summary, four metal-organic frameworks were synthesized and utilized as effective adsorbent and matrix of LDI-TOF-MS for the detection of small molecules. The obtained MOFs exhibit high desorption/ionization efficiency for mediated polar and nonpolar compounds in both positive and negative ion mode due to their favorable $\pi$-conjugated networks and plenty of oxygenated functional groups. Compared to conventional matrix, MOFs matrix has the advantages of clear matrix background, superior signal reproducibility and higher detection sensitivity in the analysis of a series of small molecules. Moreover, MOFs reveal wonderful performance in enrichment of PFCs from aqueous solution, thus enhanced MS signals of trace amount pollutants. This method also shows robust anti-interference ability to coexisting natural organic matter and 

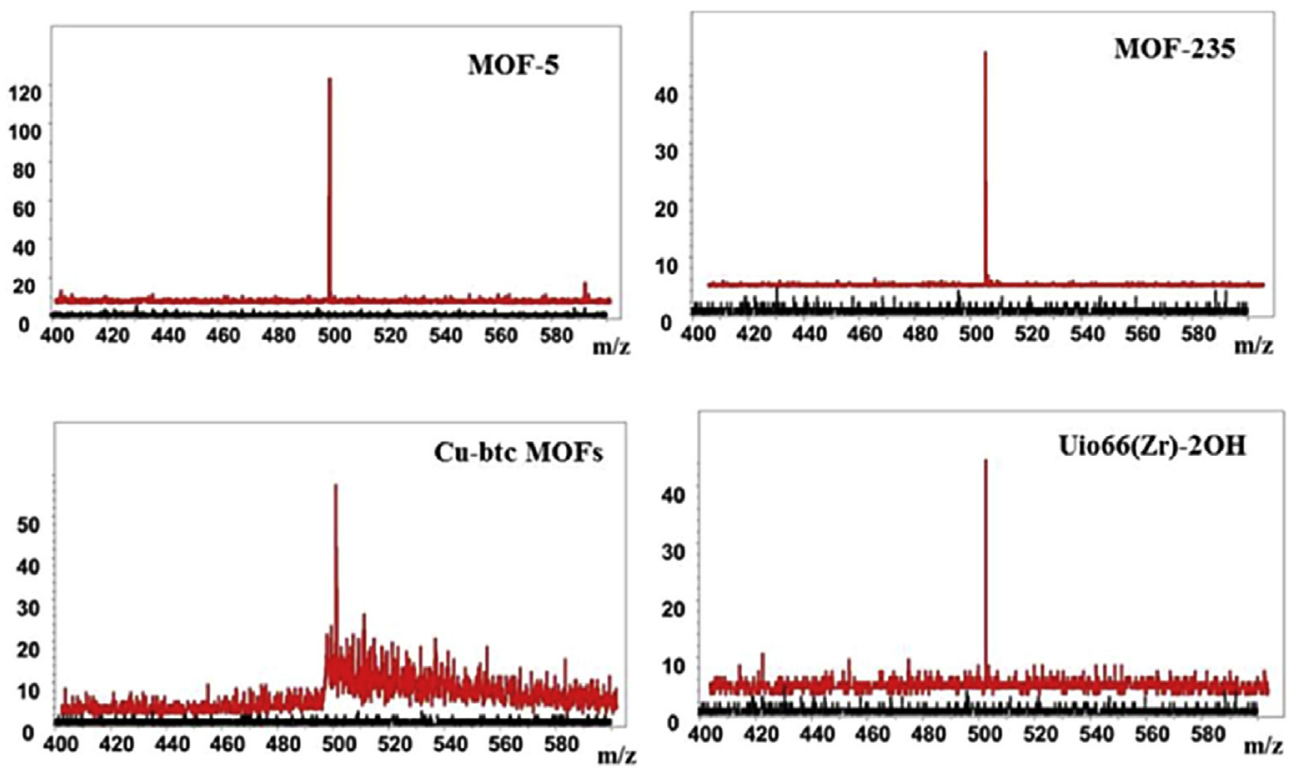

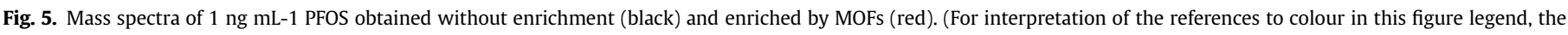
reader is referred to the web version of this article.)

protein in highly complex environmental samples. The MOFs-SPEMALDI-TOF-MS measurement is convenient, efficient and fast, so that the presented method is expected to be used in the analysis of many small molecule pollutants in the environmental field.

\section{Acknowledgments}

This work was jointly supported by National Basic Research Program of China (2015CB931903), the National Natural Science Foundation of China (21537004, 21477140, 21277152, 21277002, 21377034), and Strategic Priority Research Program of the Chinese Academy of Sciences (XDB14010201).

\section{Appendix A. Supplementary data}

Supplementary data related to this article can be found at http:// dx.doi.org/10.1016/j.micromeso.2016.10.032.

\section{References}

[1] N. Nicolaou, Y. Xu, R. Goodacre, Anal. Chem. 84 (2012) 5951-5958.

[2] M.H. Lu, Y.O. Lai, G.N. Chen, Z.W. Cai, Anal. Chem. 83 (2011) 3161-3169.

[3] Q. Liu, M. Cheng, J. Wang, G. Jiang, Chemistry 21 (2015) 5594-5599.

[4] C. Yao, C. Niu, N. Na, D. He, J. Ouyang, Anal. Chim. Acta 853 (2015) 375-383.

[5] A. Sangsuwan, B. Narupai, P. Sae-Ung, S. Rodtamai, N. Rodthongkum, V.P. Hoven, Anal. Chem. 87 (2015) 10738-10746.

[6] M. Lorey, B. Adler, H. Yan, R. Soliymani, S. Ekstrom, Anal. Chem. 87 (2015) $5255-5262$.

[7] H.P. Wu, C.J. Yu, C.Y. Lin, Y.H. Lin, W.L. Tseng, J. Am, Soc. Mass Spectrom. 20 (2009) 875-882.

[8] Z. Guo, Q.C. Zhang, H.F. Zou, B.C. Guo, J.Y. Ni, Anal. Chem. $74(2002)$ $1637-1641$.

[9] D. Cao, M. Hu, C. Han, J. Yu, L. Cui, Y. Liu, H. Wang, Y. Cai, Y. Kang, Y. Zhou, Analyst 137 (2012) 2218-2225.

[10] X. Zhang, S. Zhu, C. Deng, X. Zhang, Talanta 88 (2012) 295-302.

[11] Y. Gholipour, S.L. Giudicessi, H. Nonami, R. Erra-Balsells, Anal. Chem. 82 (2010) 5518-5526.
[12] C.Y. Lo, W.Y. Chen, C.T. Chen, Y.C. Chen, J. Proteome Res. 6 (2007) 887-893.

[13] C. Zhu, S. Guo, P. Wang, L. Xing, Y. Fang, Y. Zhai, S. Dong, Chem. Commun. 46 (2010) 7148-7150.

[14] A. Nordstrom, J.V. Apon, W. Uritboonthal, E.P. Go, G. Siuzdak, Anal. Chem. 78 (2006) 272-278.

[15] Z. Lin, J. Zheng, G. Lin, Z. Tang, X. Yang, Z. Cai, Anal. Chem. 87 (2015) 8005-8012.

[16] Q. Min, X. Zhang, X. Chen, S. Li, J.J. Zhu, Anal. Chem. 86 (2014) 9122-9130.

[17] Q. Liu, J. Shi, M. Cheng, G. Li, D. Cao, G. Jiang, Chem. Commun. 48 (2012) 1874-1876.

[18] X.S. Li, J.H. Wu, L.D. Xu, Q. Zhao, Y.B. Luo, B.F. Yuan, Y.Q. Feng, Chem. Commun. 47 (2011) 9816-9818.

[19] Y.R. Ma, X.L. Zhang, T. Zeng, D. Cao, Z. Zhou, W.H. Li, H. Niu, Y.Q. Cai, ACS Appl. Mater. interfaces. 5 (2013) 1024-1030.

[20] C. Shi, C. Deng, X. Zhang, P. Yang, ACS Appl. Mater. interfaces. 5 (2013) $7770-7776$.

[21] X.M. He, G.T. Zhu, J. Yin, Q. Zhao, B.F. Yuan, Y.Q. Feng, J. Chromatogr. A 1351 (2014) 29-36.

[22] Y. Wang, J. Xie, Y. Wu, H. Ge, X. Hu, J. Mater. Chem. A 1 (2013) 8782.

[23] Y. Peng, V. Krungleviciute, I. Eryazici, J.T. Hupp, O.K. Farha, T. Yildirim, J. Am. Chem. Soc. 135 (2013) 11887-11894.

[24] H.C. Zhou, J.R. Long, O.M. Yaghi, Chem. Rev. 112 (2012) 673-674.

[25] J.R. Li, J. Sculley, H.C. Zhou, Chem. Rev. 112 (2012) 869-932.

[26] J. Lee, O.K. Farha, J. Roberts, K.A. Scheidt, S.T. Nguyen, J.T. Hupp, Chem. Soc. Rev. 38 (2009) 1450-1459.

[27] Z. Lin, W. Bian, J. Zheng, Z. Cai, Chem. Commun. 51 (2015) 8785-8788.

[28] M. Zhao, C. Deng, X. Zhang, P. Yang, Proteomics 13 (2013) 3387-3392.

[29] Y.H. Shih, C.H. Chien, B. Singco, C.L. Hsu, C.H. Lin, H.Y. Huang, Chem. Commun. 49 (2013) 4929-4931.

[30] D.J. Tranchemontagne, J.R. Hunt, O.M. Yaghi, Tetrahedron 64 (2008) $8553-8557$.

[31] R. Wu, X. Qian, F. Yu, H. Liu, K. Zhou, J. Wei, Y. Huang, J. Mater. Chem. A 1 (2013) 11126.

[32] M. Anbia, V. Hoseini, S. Sheykhi, J. Ind. Eng. Chem. 18 (2012) 1149-1152.

[33] H.W. Tang, K.M. Ng, W. Lu, C.M. Che, Anal. Chem. 81 (2009) 4720-4729.

[34] C. Lei, K. Qian, O. Noonan, A. Nouwens, C. Yu, Nanoscale 5 (2013) $12033-12042$.

[35] Y.L. Shi, R. Vestergren, L. Xu, Z. Zhou, C.X. Li, Y. Liang, Y.Q. Cai, Environ. Sci. Technol. 50 (2016) 2396-2404.

[36] Z. Zhou, Y.L. Shi, R. Vestergren, T. Wang, Y. Liang, Y.Q. Cai, Environ. Sci. Technol. 48 (2014) 3864-3874.

[37] E. Haque, J.W. Jun, S.H. Jhung, J. Hazard. Mater. 185 (2011) 507-511. 